\title{
CORRECTION
}

\section{Correction to: A Fused Heterogeneous Deep Neural Network and Robust Feature Selection Framework for Human Actions Recognition}

Muhammad Attique Khan ${ }^{1} \cdot$ Yu-Dong Zhang ${ }^{2} \cdot$ Majed Alhaisoni ${ }^{3} \cdot$ Seifedine Kadry $^{4}$. Shui-Hua Wang ${ }^{5}$. Tanzila Saba $^{6} \cdot$ Tassawar lqbal $^{7}$

(c) King Fahd University of Petroleum \& Minerals 2022

\section{Correction to: \\ Arabian Journal for Science and Engineering https://doi.org/10.1007/s13369-021-05881-4}

In the Original publication the last name of third author in the author group was wrong. The correct last name should be "Alhaisoni". This has been updated in the original publication.

The original article can be found online at https://doi.org/10.1007/ s13369-021-05881-4.

Muhammad Attique Khan

attique.khan@hitecuni.edu.pk

Yu-Dong Zhang

yudong.zhang@leicester.ac.uk

Majed Alhaisoni

majed.alhisoni@gmail.com

Shui-Hua Wang

shuihuawang@ieee.org

1 Department of Computer Science, HITEC University, Museum Road, Taxila, Pakistan

2 Department of Informatics, University of Leicester, Leicester LE1 7RH, UK

3 College of Computer Science and Engineering, University of Ha'il, Ha'il, Saudi Arabia

4 Department of Applied Data Science, Noroff University College, Noroff Oslo, Norway

5 Department of Mathematics, University of Leicester, Lecicester LE1 7RH, UK

6 College of Computer and Information Sciences, Prince Sultan University, Riyadh, Saudi Arabia

7 Department of Computer Science, COMSATS University Islamabad, Wah Campus, Islamabad, Pakistan 